\title{
A US Population Health Survey on the Impact of COVID-19 Using the EQ-5D-5L
}

\author{
Joel W. Hay, PhD' , Cynthia L. Gong, PharmD, PhD',3 (0, Xiayu Jiao, PhD', \\ Nadine K. Zawadzki, MPH, PhDc ${ }^{7}$, Roy S. Zawadzki, BS ${ }^{4}$, A. Simon Pickard, PhD ${ }^{5}$, \\ Feng Xie, PhD ${ }^{6,7}$, Samuel A. Crawford, MS, PhDc l, and Ning Yan Gu, PhD ${ }^{6,8,9}$
}

\begin{abstract}
${ }^{1}$ Schaeffer Center for Health Policy \& Economics, School of Pharmacy, University of Southern California, Los Angeles, CA, USA; ${ }^{2} \mathrm{Fetal} \&$ Neonatal Institute, Children's Hospital Los Angeles, Los Angeles, CA, USA; ${ }^{3}$ Department of Pediatrics, Keck School of Medicine, University of Southern California, Los Angeles, CA, USA; ${ }^{4}$ Department of Statistics, Donald Bren School of Information and Computer Sciences, University of California, Irvine, Irvine, CA, USA; ${ }^{5}$ hharmacy Systems, Outcomes and Policy, University of Illinois at Chicago, Chicago, IL, USA; ${ }^{6}$ EuroQol Research Foundation, Rotterdam, the Netherlands; ${ }^{7}$ Department of Health Research Methods, Evidence and Impact (HEI), McMaster University, Hamilton, ON, Canada; ${ }^{8}$ University of San Francisco, Sacramento, CA, USA; ${ }^{N}$ YG Technologies, LLC, Santa Clarita, CA, USA.
\end{abstract}

BACKGROUND:The COVID-19 pandemic has resulted in negative impacts on the economy, population health, and health-related quality-of-life (HRQoL).

OBJECTIVE: To assess the impact of COVID-19 on US population HRGoL using the EQ-5D-5L.

DESIGN: We surveyed respondents on physical and mental health, demographics, socioeconomics, brief medical history, current COVID-19 status, sleep, dietary, financial, and spending changes. Results were compared to online and face-to-face US population norms. Predictors of EQ-5D-5L utility were analyzed using both standard and post-lasso OLS regressions. Robustness of regression coefficients against unmeasured confounding was analyzed using the E-Value sensitivity analysis.

SUBJECTS: Amazon MTurk workers $(n=2776)$ in the USA.

MAIN MEASURES: EQ-5D-5L utility and VAS scores by age group.

KEY RESULTS: We received $n=2746$ responses. Subjects 18-24 years reported lower mean (SD) health utility $(0.752(0.281))$ compared with both online $(0.844$ (0.184), $p=0.001)$ and face-to-face norms (0.919 (0.127), $p<0.001)$. Among ages 25-34, utility was worse compared to face-to-face norms only $(0.825(0.235)$ vs. $0.911(0.111)$, $p<0.001)$. For ages 35-64, utility was better during pandemic compared to online norms (0.845 (0.195) vs. 0.794 (0.247), $p<0.001)$. At age $65+$, utility values (0.827 (0.213)) were similar across all samples. VAS scores were worse for all age groups $(p<0.005)$ except ages $45-54$. Increasing age and income were correlated with increased utility, while being Asian, American Indian or Alaska Native, Hispanic, married, living alone, having history of chronic illness or self-reported depression, experiencing COVID19-like symptoms, having a family member diagnosed with COVID-19, fear of COVID-19, being underweight, and living in California were associated with worse utility scores. Results were robust to unmeasured confounding. CONCLUSIONS: HRGoL decreased during the pandemic compared to US population norms, especially for ages 18-

Received August 4, 2020

Accepted February 17, 2021

Published online March 8, 2021
24. The mental health impact of COVID-19 is significant and falls primarily on younger adults whose health outcomes may have been overlooked based on policy initiatives to date.

J Gen Intern Med 36(5):1292-301 DOI: $10.1007 / \mathrm{s} 11606-021-06674-\mathrm{z}$

(c) Society of General Internal Medicine 2021

\section{INTRODUCTION}

The COVID-19 global pandemic has resulted in exceptional societal disruption, imposing significant social, economic, and health consequences. The media has reported countless anecdotes of anxiety, depression, ${ }^{1-4}$ and domestic violence, ${ }^{5-7}$ while scientific journals have published numerous case reports and clinical studies from all over the world. ${ }^{8-10}$ As of November 24, 2020, a simple PubMed search of "covid 19 depression" yields 1,457 results, with studies using validated measures indicating increased levels of anger, depression, anxiety, and stress both within and outside the USA. With global lockdown policies of varying severities and the cancelations/closures of events, businesses, and entertainment venues, it is abundantly clear that the pandemic has significantly impacted quality-of-life of individuals worldwide, and has especially exacerbated mental health issues, including those of clinicians and healthcare workers (HCWs), who are responsible for treating mental health issues in the general public. $^{11-13}$

Missing from the COVID-19 literature in the USA is a standardized measure for health-related quality-of-life (HRQoL) that allows comparisons across different disease states and conditions such as the EQ-5D-5L, a generic health measure that is globally used in population health studies and economic evaluations because of its generalizability and ease of administration. ${ }^{14}$ It is short, simple, and validated in both online and face-to-face panels in the USA. ${ }^{15-17}$ Respondents rate mobility, self-care, usual activities, pain/discomfort, and anxiety/depression on a scale of 1-5 indicating no to extreme 
problems. Responses can be converted into health utility scores by applying a societal preference function which generates scores anchored at 0 for death and 1 for perfect health, representing a societal valuation of HRQoL; these scores can be used to calculate quality-adjusted life years (QALYs) to guide health technology assessment. Also included is a Visual Analog Scale (VAS), asking respondents to self-rate their overall health on a scale of $0-100$, thus directly reflecting HRQoL as valued by respondents. ${ }^{15}$ The EQ-5D-5L has been validated in hundreds of countries and languages, providing a standardized approach to measure and compare health within and across nations. ${ }^{15}$

Since the pandemic began, the EQ-5D-5L has been used to assess HRQoL in the Netherlands, ${ }^{18}$ Germany,${ }^{19}$ France, ${ }^{20}$ Morocco, ${ }^{21}$ China, ${ }^{22}$ Hong Kong, ${ }^{23}$ Vietnam, ${ }^{24}$ and Spain ${ }^{25}$ in various contexts including the general public, those with specific diseases, and healthcare providers; these studies have shown an overall decline in HRQoL, and pronounced worsening of anxiety and depression. The objective of this study was to assess the impact of COVID-19 on US population health using the EQ-5D-5L. Our secondary aim was to translate these findings into total lives lost by age group.

\section{METHODS}

This study used the first wave of a three-wave longitudinal panel to assess changes in HRQoL over time in the USA $(n=2,776)$. Amazon's Mechanical Turk (MTurk) platform ${ }^{26}$ was used to survey respondents on demographics, COVID-19 status, and behavior and employment changes related to COVID-19 (Supplementary material). MTurk is an online crowd-sourced platform that allows large-scale surveys to be deployed, and thus can be useful for clinical research. ${ }^{27,} 28$ Anyone $>18$ years of age can register as a respondent, known as a "Worker," to voluntarily complete "Human Intelligence Tasks" (HITs) according to criteria set by the research team, such as age or sex. HITs are activities requiring human input, such as image processing, data verification, surveys, and data processing (e.g., translation, transcribing audio content). ${ }^{26}$ Because the platform is online, all tasks require an active internet connection. In our study, all Workers were eligible as long as they resided in the USA, and were given $\$ 2$ compensation for their time. Our only inclusion criteria were to ensure that age and gender across respondents was similar to US census data.

We surveyed respondents on HRQoL using the EQ-5D$5 \mathrm{~L}$. We also included questions on demographics, brief medical history, socioeconomic status, current COVID-19 status, and employment status. Finally, we asked respondents to rate their fear of COVID-19's impact on their health and financial situations on a scale of $0-10$, whether respondents were under mandatory social distancing, and respondents' level of support for social distancing policies on a scale of $0-10$. We assessed the quality and demographics of responses throughout the data collection process to ensure a relatively even distribution of sample respondents across age and gender similar to the general US population (Table 1). Criteria restricting respondents to those aged 55+ was added after the initial 2000 subjects were recruited to improve the age representativeness of the sample.

Demographics were compared to the general population based on US census data. Utility values were calculated from EQ-5D-5L responses using US-derived value weights. ${ }^{17} \mathrm{We}$ compared EQ-5D-5L results with prepandemic results we collected prior to COVID-19 $(n=40)$ and with US population norms from previously derived online $(n=2,018)$ as well as face-to-face $(n=1,134)$ interviews. ${ }^{16} \mathrm{We}$ used $t$-tests and chi-square tests for numeric and categorical variables respectively to identify statistically significant differences.

We employed standard ordinary least squares (OLS) regression to evaluate the impact of demographic characteristics, personal C19 symptoms, diagnosis of COVID-19 in family, knowing someone with a COVID-19 diagnosis, and fear of health and financial consequences (full list, Table 3) on EQ-5D-5L utility score. To improve model fit, we included additional predictors such as BMI category, disease history (hypercholesterolemia, hypertension, arthritis, diabetes, heart failure, stroke, bronchitis, asthma, self-reported depression, migraine, and cancer), and US state of residence, as well as all two-way interactions between predictors. Because of the high number of predictors relative to number of responses, we then employed lasso regression, a supervised machine learning algorithm that reduces overfitting and multicollinearity. ${ }^{29} \mathrm{We}$ constrained lasso from regularizing the characteristics of interest listed above and employed 10-fold cross-validation to minimize mean squared error (MSE). Details are described in the Technical Appendix.

Of the variables not constrained from regularization, lasso selected three predictors and two interactions: arthritis, diabetes, self-reported depression, stroke interacted with fear of COVID-19's impact on health (1-10 scale), and underweight BMI interacted with residing in California. Since lasso coefficients are biased and not intended for inference, ${ }^{30}$ we fit a second OLS model to obtain unbiased coefficients on these selected predictors (full variable list, Table 4).

For inference, the coefficients given by the second (post-lasso) OLS regression were bootstrapped 500 iterations to estimate standard errors, computed as the standard deviation of the bootstrap replicates. ${ }^{30}$ The standard errors were then used to construct normal-theory $97.5 \%$ confidence intervals for the regression coefficients, using the Bonferroni correction to compare the results of the two OLS models. We report the median bootstrap estimates as the post-lasso OLS point estimates with the normal-theory 
Table 1 Sample Demographics vs. US Population

\begin{tabular}{|c|c|c|c|}
\hline & $\begin{array}{l}\text { Sample, } n \\
(\%) \\
N=2,746\end{array}$ & $\begin{array}{l}\text { US } \\
\text { population } \\
(\%)\end{array}$ & Reference \\
\hline $\begin{array}{l}\text { Age (median, } \\
\text { vears) }\end{array}$ & 39 & 38.3 & \multirow{7}{*}{$\begin{array}{l}\text { US Census } \\
\text { Bureau }^{42} \\
\text { US Census }_{\text {Bureau }^{42}}\end{array}$} \\
\hline Gender & & & \\
\hline Male & $1342(48.9)$ & 49.1 & \\
\hline Female & 1365 (49.7) & 50.8 & \\
\hline Transgender & $13(0.5)$ & & \\
\hline Other/prefer not & $17(0.6)$ & NA & \\
\hline to say & $9(0.3)$ & & \\
\hline Race & & & $\begin{array}{l}\text { US Census } \\
\text { Bureau }^{43}\end{array}$ \\
\hline White & $1,888(68.8)$ & 76.5 & \\
\hline Black & $200(7.3)$ & 13.4 & \\
\hline Asian & $188(6.9)$ & 5.9 & \\
\hline Native & $4(0.2)$ & 0.2 & \\
\hline \multicolumn{4}{|l|}{$\begin{array}{l}\text { Hawaiian/Pacific } \\
\text { Islander }\end{array}$} \\
\hline Multi-race & $405(14.8)$ & 2.7 & \\
\hline Other & $26(1.0)$ & NA & \\
\hline $\begin{array}{l}\text { American Indian } \\
\text { or Alaska Native }\end{array}$ & $17(0.6)$ & 1.3 & \\
\hline Hispanic Ethnicity & & 18.3 & $\begin{array}{l}\text { US Census } \\
\text { Bureau }^{43}\end{array}$ \\
\hline Yes & $272(9.9)$ & & \\
\hline No & $2,452(89.3)$ & & \\
\hline Prefer not to say & $20(0.7)$ & & \\
\hline Missing & $2(0.1)$ & & \\
\hline Education & $2(0.1)$ & & \multirow[t]{7}{*}{$\begin{array}{l}\text { US Census } \\
\text { Bureau }^{44}\end{array}$} \\
\hline $\begin{array}{l}\text { Less than high } \\
\text { school degree }\end{array}$ & $14(0.5)$ & 10.6 & \\
\hline $\begin{array}{l}\text { High school } \\
\text { degree or } \\
\text { equivalent }\end{array}$ & $264(9.6)$ & 28.3 & \\
\hline $\begin{array}{l}\text { Some college but } \\
\text { no degree }\end{array}$ & 457 (16.6) & 18 & \\
\hline Associate degree & $316(11.5)$ & 9.8 & \\
\hline Bachelor degree & $1,212(44.1)$ & 21.3 & \\
\hline Graduate degree & $483(17.6)$ & 12 & \\
\hline Marital status & & & \multirow[t]{7}{*}{$\begin{array}{l}\text { US Census } \\
\text { Bureau }^{45}\end{array}$} \\
\hline Single & $1,073(39.1)$ & 33.8 & \\
\hline Married & $1,284(46.8)$ & 47.8 & \\
\hline Separated & $23(0.8)$ & 1.9 & \\
\hline Divorced & $266(9.7)$ & 10.9 & \\
\hline Widowed & $75(2.7)$ & 5.7 & \\
\hline Prefer not to say & $25(0.9)$ & & \\
\hline Income & & & \multirow[t]{12}{*}{$\begin{array}{l}\text { US Census } \\
\text { Bureau }^{46}\end{array}$} \\
\hline $\begin{array}{l}\text { Less than } \\
\$ 20,000\end{array}$ & $278(10.1)$ & 14.7 & \\
\hline$\$ 20,000$ to & $425(15.5)$ & 13.2 & \\
\hline$\$ 34,999$ & & & \\
\hline $\begin{array}{l}\$ 35,000 \text { to } \\
\$ 49,999\end{array}$ & $482(17.6)$ & 12 & \\
\hline $\begin{array}{l}\$ 49,999 \\
\$ 50,000 \text { to }\end{array}$ & $694(25.3)$ & 17.2 & \\
\hline$\$ 74,999$ & & & \\
\hline$\$ 75,000$ to & $441(16.1)$ & 12.5 & \\
\hline $\begin{array}{l}\$ 99,999 \\
\$ 100,000 \text { to }\end{array}$ & & & \\
\hline $\begin{array}{l}\$ 100,000 \text { to } \\
\$ 149,999\end{array}$ & $306(11.1)$ & 14.9 & \\
\hline Over $\$ 150,000$ & $118(4.3)$ & 15.5 & \\
\hline Missing & $2(0.1)$ & & \\
\hline \multicolumn{4}{|l|}{ Insurance } \\
\hline Private & $377(13.8)$ & 55.1 & \multirow{4}{*}{$\begin{array}{l}\text { Kaiser Family } \\
\text { Foundation }{ }^{47} \\
\text { US Census } \\
\text { Bureau }^{48}\end{array}$} \\
\hline Medicare & $114(4.2)$ & 17.4 & \\
\hline Medicaid & $83(3.0)$ & 17.9 & \\
\hline ACA & $64(2.3)$ & 3.3 & \\
\hline
\end{tabular}

(continued on next page)
Table 1. (continued)

\begin{tabular}{|c|c|c|c|}
\hline & $\begin{array}{l}\text { Sample, } n \\
(\%) \\
N=2,746\end{array}$ & $\begin{array}{l}\text { US } \\
\text { population } \\
(\%)\end{array}$ & Reference \\
\hline Self-pay & $30(1.1)$ & 10.8 & \\
\hline None & $119(4.3)$ & 8.5 & \\
\hline Don’t know & $7(0.3)$ & NA & \\
\hline Missing & $1,952(71.1)$ & & \\
\hline Political & & & Gallup Poll ${ }^{49}$ \\
\hline Affiliation & & & \\
\hline Republican & $778(28.3)$ & 27 & \\
\hline Democrat & $1,248(45.5)$ & 31 & \\
\hline Independent & $631(23.0)$ & 39 & \\
\hline None & $20(0.7)$ & NA & \\
\hline Missing & $69(2.5)$ & & \\
\hline Live Alone & & & $\begin{array}{l}\text { US Census } \\
\text { Bureau }^{50}\end{array}$ \\
\hline Yes & 592 (21.6) & 26 & \\
\hline No & $1,694(61.7)$ & 74 & \\
\hline Missing & $460(16.8)$ & & \\
\hline Medical History & & & \\
\hline Cholesterol & 408 (14.9) & 11.8 & $\mathrm{CDC}^{51}$ \\
\hline Hypertension & $438(16.0)$ & 33.2 & $\mathrm{CDC}_{53}^{52}$ \\
\hline Arthritis & $278(10.1)$ & 23.7 & $\mathrm{CDC}^{53}$ \\
\hline Diabetes & $214(7.8)$ & 10.5 & $\mathrm{CDC}^{54}$ \\
\hline Heart failure & $56(2.0)$ & 2.4 & $\mathrm{CDC}^{55}$ \\
\hline Stroke & $61(2.2)$ & 3.1 & $\mathrm{CDC}^{56}$ \\
\hline Bronchitis & $155(5.6)$ & 3.6 & $\mathrm{CDC}^{57}$ \\
\hline Asthma & 329 (12.0) & 7.7 & $\mathrm{CDC}^{58}$ \\
\hline Depression & $599(21.8)$ & 7.6 & $\mathrm{CDC}^{59}$ \\
\hline Migraine & $248(9.0)$ & 15.9 & $\mathrm{CDC}^{60}$ \\
\hline Cancer & $268(9.8)$ & 9.4 & $\mathrm{CDC}^{61}$ \\
\hline Missing & $198(7.2)$ & & \\
\hline Tobacco Use & & & $\mathrm{CDC}^{62,63}$ \\
\hline Current & 368 (13.4) & 14 & \\
\hline Previous & $686(25.0)$ & 21.3 & \\
\hline Never & $1,148(41.8)$ & 64.7 & \\
\hline Missing & $543(19.8)$ & & \\
\hline BMI & & & $\begin{array}{l}\text { DQYDJ }^{64} ; \\
\text { CDC }^{65}\end{array}$ \\
\hline$<18.5$ & $163(5.9)$ & 1.6 & \\
\hline $18.5-24.9$ & $881(32.1)$ & 27.5 & \\
\hline $25.0-29.9$ & $652(23.8)$ & 31.6 & \\
\hline$>30$ & $507(18.5)$ & 39.4 & \\
\hline Missing & $543(19.8)$ & & \\
\hline Employment & & & $\begin{array}{l}\text { US Census } \\
\text { Bureau }^{66}\end{array}$ \\
\hline Full-time & $1605(58.5)$ & 59.8 & \\
\hline Part-time & $439(16.0)$ & & \\
\hline $\begin{array}{l}\text { Unemployed } \\
\text { seeking }\end{array}$ & $174(6.3)$ & 4.9 & \\
\hline $\begin{array}{l}\text { Unemployed not } \\
\text { seeking }\end{array}$ & $105(3.8)$ & & \\
\hline Student & $64(2.3)$ & & \\
\hline Retired & $225(8.1)$ & & \\
\hline Disability & $34(1.2)$ & & \\
\hline Homemaker & $87(3.2)$ & & \\
\hline Don't know & $4(0.2)$ & & \\
\hline Missing & $9(0.3)$ & & \\
\hline
\end{tabular}

bootstrap intervals. A partial $F$-test was employed to determine whether the post-lasso model significantly fits the data better than the standard model.

Since this is a non-randomized study, there is potential for unmeasured confounding to bias our estimated associations between respondent characteristics and quality-of-life. We therefore calculated regression coefficient $E$-values to assess the required strength that any unmeasured confounder must have to nullify our model's statistically significant findings 
Table 2 Comparison of EQ-5D-5L Utility Values and VAS Scores to Norms

\begin{tabular}{|c|c|c|c|c|c|c|c|}
\hline \multicolumn{8}{|c|}{ EQ-5D-5L mean utility values } \\
\hline Age & $\begin{array}{l}\text { During } \\
(n=2,746)\end{array}$ & $(n=40)$ & $p$ value & $(n=2,018)$ & $p$ value & $(n=1,134)$ & $p$ value \\
\hline $25-34$ & 0.825 & 0.860 & 0.490 & 0.811 & 0.305 & 0.911 & 0.000 \\
\hline $35-44$ & 0.845 & 0.867 & 0.393 & 0.794 & 0.001 & 0.841 & 0.806 \\
\hline $45-54$ & 0.818 & 0.736 & 0.452 & 0.760 & 0.001 & 0.816 & 0.969 \\
\hline Age & $\begin{array}{l}\text { During } \\
(n=2,746)\end{array}$ & $\begin{array}{l}\text { Pre } \\
(n=40)\end{array}$ & $p$ value & $\begin{array}{l}\text { Online } \\
(n=2,018)\end{array}$ & $p$ value & $\begin{array}{l}\text { F2F } \\
(n=1,134)\end{array}$ & $p$ value \\
\hline $18-24$ & 73.1 & 72.3 & 0.950 & 79.9 & 0.001 & 84.9 & 0.000 \\
\hline $25-34$ & 76.6 & 60.8 & 0.008 & 77.7 & 0.261 & 84.4 & 0.000 \\
\hline $35-44$ & 74.2 & 74.9 & 0.894 & 74.7 & 0.686 & 78.1 & 0.004 \\
\hline $45-54$ & 73.2 & 70.5 & 0.709 & 71.1 & 0.172 & 75.9 & 0.101 \\
\hline $55-64$ & 73.4 & 71.0 & 0.827 & 71.5 & 0.194 & 78.8 & 0.002 \\
\hline
\end{tabular}

Utility values represent societal preference scores for the health states as rated by respondents and can be used to calculate quality-adjusted life years (QALYS). VAS scores directly reflect the respondents' valuation of his/her own health status. Bold values indicate statistical significance ( $p<0.05)$

(see Technical Appendix). ${ }^{31,32} \mathrm{R}$ version 4.0.1 was used to perform statistical analyses with significance set at 0.05 .

Finally, we estimated QALY gain/loss by age group compared to population norms. We calculated the utility change compared to norms by age group, then multiplied this change by the total population in each age group to obtain populationwide change in utility. This calculation assumed that any detected utility change lasts 12 months. We then divided the total QALY gain/loss by the estimated life expectancy for each age group to extrapolate total lives lost resulting from changes in HRQoL captured by the EQ-5D-5L.

\section{RESULTS}

\section{Sample}

We received 2,746 complete responses to the EQ-5D-5L. Compared with the US general population, our sample was slightly older, with higher education and income, less Hispanic and Black respondents, but more individuals identifying as multi-race. There was also less chronic hypertension, diabetes, arthritis, and migraine, but more hypercholesterolemia, depression, asthma, and bronchitis (cancer). Full-time employment, gender, age, marital status, and BMI $\geq 30$ were similar to the general US population (Table 1).

Most respondents reported working in management (9.6\%), business/finance $(11.9 \%)$, computer and mathematical industries $(11.3 \%)$, and office/administrative support (10.3\%). Less than $1 \%$ reported working in protective services, grounds maintenance, farming/fishing/forestry, or the military. As a result of COVID-19, 52.8\% reported no change in their employment, $31.9 \%$ reported working at home, $5.8 \%$ reported losing their jobs, and $9.6 \%$ reported being temporarily laid off. $8.8 \%$ reported that COVID-19 completely prevented them from working. Most (70.4\%) reported no hours of missed work due to COVID-19.
When rating fear of COVID-19's impact on their health, $59.5 \%$ of the sample reported a score of $>5$ on a scale of $0-10$ (mean 5.20, SD 2.95). When rating fear of COVID-19's impact on their economic/financial well-being, $67.6 \%$ reported a score of $\geq 5$ (mean 5.79, SD 3.01). $90.8 \%$ of respondents were under mandatory social distancing, and $90.6 \%$ scored $\geq 5$ (mean 8.37, SD 2.5) in support of social distancing policies to prevent the spread of COVID-19.

\section{$E Q-5 D-5 L$}

Among ages 18-24 ( $n=198)$, the mean (SD) utility value was $0.752(0.281)$, significantly lower compared to pre-pandemic (0.921 (0.124), $p=0.01)$, online (0.844 (0.184), $p<0.001)$, and face-to-face EQ-5D-5L norms (0.919 (0.127), $p<0.001)$. Among ages 25-34 $(n=817)$, utility was significantly worse compared to face-to-face norms $(0.825$ (0.235) vs. 0.911 (0.111), $p<0.001)$; no significant differences were seen vs. online norms. Among ages 35-64 $(n=1,488)$, utility values were higher during-pandemic but only vs. online norms; there were no significant differences compared to pre-pandemic and face-to-face samples. At age $65+(n=248)$, utility values $(0.827$ (0.213)) were nearly identical across all samples.

For the VAS, all age groups except age 45-54 had significantly worse scores compared to face-to-face norms. Only ages 18-24 reported significantly worse mean VAS scores compared to online norms ( 73.1 vs. $79.9, p=0.001$ ), and ages 25-34 reported significantly better scores compared to prepandemic (76.6 vs. 60.8, $p=0.008)$. Pre-pandemic sample sizes for other age groups were too small $(n<5)$ to draw meaningful inferences. All EQ-5D-5L and VAS comparisons between the MTurk sample and online and face-to-face samples are stratified by age group in Table 2 .

Differences appear to be driven by the anxiety/depression dimension of the EQ-5D-5L, which was worse during pandemic vs. either norm (Fig. 1). This was especially pronounced among females and "other" gendered persons 
Table 3 Relationship Between EQ-5D Utility Score and Select Respondent Characteristics, Estimated by OLS Regression

\begin{tabular}{|c|c|c|c|c|c|c|c|}
\hline Predictor & Estimate & $\begin{array}{l}\text { Lower } \\
97.5 \% \text { CI }\end{array}$ & $\begin{array}{l}\text { Upper } \\
97.5 \% \text { CI }\end{array}$ & Std. error & $P$ value* & $E$-value & $\begin{array}{l}E \text {-value } 95 \% \\
\text { CL }\end{array}$ \\
\hline (Intercept) & 0.801 & 0.649 & 0.952 & 0.067 & $<0.001$ & 54.162 & 31.309 \\
\hline Gender, male & (Reference) & (Reference) & (Reference) & (Reference) & (Reference) & (Reference) & (Reference) \\
\hline Gender, female & -0.011 & -0.032 & 0.010 & 0.009 & 0.246 & 1.267 & 1.000 \\
\hline Gender, prefer not to say & -0.095 & -0.245 & 0.055 & 0.067 & 0.157 & 2.324 & 1.000 \\
\hline Gender, other & -0.203 & -0.363 & -0.044 & 0.071 & 0.004 & 4.055 & 1.932 \\
\hline Age group, 18-24 & (Reference) & (Reference) & (Reference) & (Reference) & (Reference) & (Reference) & (Reference) \\
\hline Age group, 25-34 & 0.063 & 0.019 & 0.106 & 0.019 & 0.001 & 1.918 & 1.467 \\
\hline Age group, 35-44 & 0.078 & 0.033 & 0.124 & 0.020 & $<0.001$ & 2.104 & 1.626 \\
\hline Age group, 45-54 & 0.058 & 0.008 & 0.109 & 0.023 & 0.010 & 1.857 & 1.297 \\
\hline Age group, 55-64 & 0.063 & 0.014 & 0.112 & 0.022 & 0.004 & 1.918 & 1.391 \\
\hline Age group, $\geq 65$ & 0.083 & 0.029 & 0.138 & 0.024 & 0.001 & 2.168 & 1.592 \\
\hline Race, White & (Reference) & (Reference) & (Reference) & (Reference) & (Reference) & (Reference) & (Reference) \\
\hline $\begin{array}{l}\text { Race, American Indian or Alaska } \\
\text { Native }\end{array}$ & 0.109 & -0.015 & 0.234 & 0.055 & 0.048 & 2.513 & 1.083 \\
\hline Race, Asian & 0.045 & 0.006 & 0.085 & 0.018 & 0.010 & 1.700 & 1.249 \\
\hline Race, Black or African American & -0.004 & -0.043 & 0.034 & 0.017 & 0.807 & 1.147 & 1.000 \\
\hline Race, multiple-race & -0.055 & -0.120 & 0.010 & 0.029 & 0.058 & 1.821 & 1.000 \\
\hline Race, Native Hawaiian or Other & -0.205 & -0.446 & 0.036 & 0.107 & 0.057 & 4.094 & 1.000 \\
\hline Pacific Islander & & & & & & & \\
\hline Race, other & 0.084 & -0.017 & 0.184 & 0.045 & 0.061 & 2.181 & 1.000 \\
\hline Race, prefer not to say & 0.114 & -0.039 & 0.268 & 0.068 & 0.095 & 2.583 & 1.000 \\
\hline Hispanic ethnicity, no & (Reference) & (Reference) & (Reference) & (Reference) & (Reference) & (Reference) & (Reference) \\
\hline Hispanic ethnicity, prefer not to say & 0.002 & -0.137 & 0.141 & 0.062 & 0.974 & 1.100 & 1.000 \\
\hline Hispanic ethnicity, yes & -0.056 & -0.093 & -0.018 & 0.017 & 0.001 & 1.833 & 1.427 \\
\hline Marital status, single & (Reference) & (Reference) & (Reference) & (Reference) & (Reference) & (Reference) & (Reference) \\
\hline Marital status, divorced & -0.004 & -0.044 & 0.035 & 0.018 & 0.807 & 1.147 & 1.000 \\
\hline Marital status, married & -0.045 & -0.072 & -0.018 & 0.012 & $<0.001$ & 1.700 & 1.412 \\
\hline Marital status, prefer not to say & 0.035 & -0.085 & 0.154 & 0.053 & 0.513 & 1.579 & 1.000 \\
\hline Marital status, separated & -0.042 & -0.148 & 0.064 & 0.047 & 0.374 & 1.664 & 1.000 \\
\hline Marital status, widowed & -0.004 & -0.068 & 0.060 & 0.029 & 0.881 & 1.147 & 1.000 \\
\hline Annual income, less than $\$ 20,000$ & (Reference) & (Reference) & (Reference) & (Reference) & (Reference) & (Reference) & (Reference) \\
\hline Annual income, $\$ 20,000$ to $\$ 34,999$ & 0.032 & -0.011 & 0.074 & 0.019 & 0.094 & 1.543 & 1.000 \\
\hline Annual income, $\$ 35,000$ to $\$ 49,999$ & 0.050 & 0.008 & 0.092 & 0.019 & 0.008 & 1.760 & 1.294 \\
\hline Annual income, $\$ 50,000$ to $\$ 74,999$ & 0.085 & 0.044 & 0.125 & 0.018 & $<0.001$ & 2.193 & 1.758 \\
\hline Annual income, $\$ 75,000$ to $\$ 99,999$ & 0.077 & 0.033 & 0.120 & 0.020 & $<0.001$ & 2.092 & 1.614 \\
\hline Annual income, $\$ 100,000$ to $\$ 149,999$ & 0.097 & 0.048 & 0.145 & 0.022 & $<0.001$ & 2.350 & 1.808 \\
\hline Annual income, over $\$ 150,000$ & 0.146 & 0.084 & 0.207 & 0.028 & $<0.001$ & 3.057 & 2.274 \\
\hline $\begin{array}{l}\text { Education, less than high school } \\
\text { degree }\end{array}$ & (Reference) & (Reference) & (Reference) & (Reference) & (Reference) & (Reference) & (Reference) \\
\hline $\begin{array}{l}\text { Education, High school degree or } \\
\text { equivalent (e.g., GED) }\end{array}$ & 0.050 & -0.099 & 0.199 & 0.066 & 0.451 & 1.760 & 1.000 \\
\hline Education, some college but no degree & 0.027 & -0.120 & 0.174 & 0.066 & 0.679 & 1.481 & 1.000 \\
\hline Education, associate degree & 0.035 & -0.114 & 0.183 & 0.066 & 0.601 & 1.579 & 1.000 \\
\hline Education, bachelor degree & 0.024 & -0.123 & 0.170 & 0.065 & 0.718 & 1.443 & 1.000 \\
\hline Education, graduate degree & 0.031 & -0.117 & 0.180 & 0.066 & 0.633 & 1.531 & 1.000 \\
\hline Live alone & -0.033 & -0.061 & -0.005 & 0.012 & 0.008 & 1.555 & 1.244 \\
\hline $\begin{array}{l}\text { Experienced COVID-19-like symp- } \\
\text { toms not serious enough to require } \\
\text { hospitalization }\end{array}$ & -0.039 & -0.074 & -0.003 & 0.016 & 0.014 & 1.628 & 1.215 \\
\hline $\begin{array}{l}\text { Has a family member diagnosed with } \\
\text { COVID-19 }\end{array}$ & -0.090 & -0.138 & -0.042 & 0.021 & $<0.001$ & 2.258 & 1.747 \\
\hline $\begin{array}{l}\text { Knows someone with a COVID-19 } \\
\text { diagnosis }\end{array}$ & -0.012 & -0.039 & 0.014 & 0.012 & 0.294 & 1.282 & 1.000 \\
\hline $\begin{array}{l}\text { Fear of COVID-19's impact on health } \\
\text { (1-10 scale) }\end{array}$ & -0.013 & -0.017 & -0.009 & 0.002 & $<0.001$ & 1.296 & 1.238 \\
\hline $\begin{array}{l}\text { Fear of COVID-19's impact on finan- } \\
\text { ces }(1-10 \text { scale })\end{array}$ & -0.002 & -0.006 & 0.002 & 0.002 & 0.191 & 1.100 & 1.000 \\
\hline
\end{tabular}

Abbreviations: OLS ordinary least squares, CI confidence interval, CL confidence limit, BMI body mass index, COVID-19 coronavirus disease 2019 *Significance level: 0.025

(Supplemental Figure 1). When stratified by BMI, those who were underweight or obese experienced the most severe/ extreme anxiety/depression (Supplemental Figure 2).

\section{Predictors of EQ-5D-5L Utility}

Table 3 displays the standard OLS regression results along with $E$-values for the point estimates and their confidence interval limits closer to the null. Compared to males, "other" gendered persons have significantly lower utility scores, whereas females and "prefer not to say" gendered persons differ non-significantly from males. Being $25+$ years old was significantly associated $(p \leq 0.025)$ with higher EQ-5D-5L utility relative to ages $18-24$. Asian, American Indian, or Alaska Native race was significantly associated with lower utility compared to being White; other race groups differed nonsignificantly from Whites. Hispanic ethnicity was also significantly associated with lower utility, as was being married, 
Table 4 Relationship Between EQ-5D Utility Score and Select Respondent Characteristics, Estimated by OLS Regression Following Lasso

\begin{tabular}{|c|c|c|c|c|c|c|}
\hline Predictor & Estimate* & $\begin{array}{l}\text { Lower } 97.5 \% \\
\text { CI* }\end{array}$ & $\begin{array}{l}\text { Upper } 97.5 \% \\
\text { CI* }\end{array}$ & Std. error* & $E$-value & $\begin{array}{l}E \text {-value 95\% } \\
\text { CL }\end{array}$ \\
\hline (Intercept) & 0.851 & 0.709 & 0.975 & 0.059 & 66.702 & 41.220 \\
\hline Gender, male & (Reference) & (Reference) & (Reference) & (Reference) & (Reference) & (Reference) \\
\hline Gender, female & 0.002 & -0.018 & 0.021 & 0.009 & 1.100 & 1.000 \\
\hline Gender, prefer not to say & -0.082 & -0.186 & 0.030 & 0.048 & 2.155 & 1.000 \\
\hline Gender, other & -0.190 & -0.445 & 0.083 & 0.118 & 3.808 & 1.000 \\
\hline Age group, 18-24 & (Reference) & (Reference) & (Reference) & (Reference) & (Reference) & (Reference) \\
\hline Age group, 25-34 & 0.060 & 0.015 & 0.108 & 0.021 & 1.881 & 1.378 \\
\hline Age group, 35-44 & 0.071 & 0.025 & 0.120 & 0.021 & 2.017 & 1.517 \\
\hline Age group, 45-54 & 0.055 & 0.004 & 0.109 & 0.023 & 1.821 & 1.252 \\
\hline Age group, 55-64 & 0.066 & 0.019 & 0.117 & 0.022 & 1.955 & 1.430 \\
\hline Age group, $\geq 65$ & 0.092 & 0.034 & 0.147 & 0.025 & 2.284 & 1.677 \\
\hline Race, White & (Reference) & (Reference) & (Reference) & (Reference) & (Reference) & (Reference) \\
\hline Race, American Indian or Alaska Native & 0.099 & 0.013 & 0.190 & 0.040 & 2.377 & 1.402 \\
\hline Race, Asian & 0.025 & -0.013 & 0.063 & 0.017 & 1.456 & 1.000 \\
\hline Race, Black or African American & -0.016 & -0.060 & 0.026 & 0.019 & 1.338 & 1.000 \\
\hline Race, multiple-race & -0.030 & -0.081 & 0.027 & 0.024 & 1.518 & 1.000 \\
\hline Race, Native Hawaiian or Other Pacific Islander & -0.157 & -0.520 & 0.217 & 0.164 & 3.234 & 1.000 \\
\hline Race, other & 0.049 & -0.042 & 0.132 & 0.039 & 1.748 & 1.000 \\
\hline Race, prefer not to say & 0.068 & -0.026 & 0.165 & 0.042 & 1.980 & 1.000 \\
\hline Hispanic ethnicity, no & (Reference) & (Reference) & (Reference) & (Reference) & (Reference) & (Reference) \\
\hline Hispanic ethnicity, prefer not to say & 0.013 & -0.064 & 0.092 & 0.035 & 1.296 & 1.000 \\
\hline Hispanic ethnicity, yes & -0.043 & -0.081 & 0.001 & 0.018 & 1.676 & 1.216 \\
\hline Marital status, single & (Reference) & (Reference) & (Reference) & (Reference) & (Reference) & (Reference) \\
\hline Marital status, divorced & -0.008 & -0.045 & 0.030 & 0.017 & 1.220 & 1.000 \\
\hline Marital status, married & -0.044 & -0.072 & -0.017 & 0.012 & 1.688 & 1.399 \\
\hline Marital status, prefer not to say & 0.018 & -0.097 & 0.117 & 0.048 & 1.365 & 1.000 \\
\hline Marital status, separated & -0.020 & -0.145 & 0.098 & 0.054 & 1.392 & 1.000 \\
\hline Marital status, widowed & 0.001 & -0.057 & 0.062 & 0.026 & 1.069 & 1.000 \\
\hline Annual income, less than $\$ 20,000$ & (Reference) & (Reference) & (Reference) & (Reference) & (Reference) & (Reference) \\
\hline Annual income, $\$ 20,000$ to $\$ 34,999$ & 0.021 & -0.020 & 0.064 & 0.019 & 1.405 & 1.000 \\
\hline Annual income, $\$ 35,000$ to $\$ 49,999$ & 0.024 & -0.017 & 0.066 & 0.018 & 1.443 & 1.000 \\
\hline Annual income, $\$ 50,000$ to $\$ 74,999$ & 0.065 & 0.031 & 0.105 & 0.017 & 1.943 & 1.540 \\
\hline Annual income, $\$ 75,000$ to $\$ 99,999$ & 0.058 & 0.017 & 0.102 & 0.019 & 1.857 & 1.403 \\
\hline Annual income, $\$ 100,000$ to $\$ 149,999$ & 0.072 & 0.033 & 0.115 & 0.018 & 2.029 & 1.601 \\
\hline Annual income, over $\$ 150,000$ & 0.115 & 0.076 & 0.162 & 0.019 & 2.597 & 2.102 \\
\hline Education, less than high school degree & (Reference) & (Reference) & (Reference) & (Reference) & (Reference) & (Reference) \\
\hline $\begin{array}{l}\text { Education, high school degree or equivalent (e.g., } \\
\text { GED) }\end{array}$ & 0.042 & -0.082 & 0.180 & 0.058 & 1.664 & 1.000 \\
\hline Education, some college but no degree & 0.026 & -0.097 & 0.165 & 0.058 & 1.469 & 1.000 \\
\hline Education, associate degree & 0.041 & -0.088 & 0.175 & 0.059 & 1.652 & 1.000 \\
\hline Education, bachelor degree & 0.014 & -0.108 & 0.149 & 0.057 & 1.311 & 1.000 \\
\hline Education, graduate degree & 0.024 & -0.098 & 0.160 & 0.058 & 1.443 & 1.000 \\
\hline Live alone & -0.035 & -0.062 & -0.007 & 0.012 & 1.579 & 1.275 \\
\hline $\begin{array}{l}\text { Experienced COVID-19-like symptoms not seri- } \\
\text { ous enough to require hospitalization }\end{array}$ & -0.023 & -0.055 & 0.013 & 0.015 & 1.431 & 1.000 \\
\hline Has a family member diagnosed with COVID-19 & -0.080 & -0.145 & -0.023 & 0.027 & 2.130 & 1.483 \\
\hline Knows someone with a COVID-19 diagnosis & -0.005 & -0.033 & 0.020 & 0.012 & 1.167 & 1.000 \\
\hline $\begin{array}{l}\text { Fear of COVID-19's impact on health (1-10 } \\
\text { scale) }\end{array}$ & -0.010 & -0.013 & -0.006 & 0.002 & 1.252 & 1.187 \\
\hline $\begin{array}{l}\text { Fear of COVID-19's impact on finances (1-10 } \\
\text { scale) }\end{array}$ & -0.002 & -0.005 & 0.002 & 0.002 & 1.100 & 1.000 \\
\hline Arthritis & -0.115 & -0.151 & -0.077 & 0.017 & 2.597 & 2.152 \\
\hline Diabetes & -0.081 & -0.126 & -0.036 & 0.020 & 2.142 & 1.663 \\
\hline Depression & -0.122 & -0.147 & -0.097 & 0.011 & 2.696 & 2.397 \\
\hline $\begin{array}{l}\text { Fear of COVID-19's impact on health (1-10 } \\
\text { scale) * stroke }\end{array}$ & -0.034 & -0.062 & -0.008 & 0.012 & 1.567 & 1.260 \\
\hline BMI category, underweight $*$ California & -0.263 & -0.415 & -0.109 & 0.068 & 5.375 & 2.813 \\
\hline
\end{tabular}

Abbreviations: OLS ordinary least squares, CI confidence interval, CL confidence limit, BMI body mass index, COVID-19 Coronavirus disease 2019 *The coefficients given by this (post-lasso) OLS regression were bootstrapped to estimate standard errors, computed as the standard deviation of the bootstrap replicates. The standard errors were then used to construct Bonferroni-corrected normal-theory confidence intervals for the regression coefficients. In this table, we report the median bootstrap estimates as the model point estimates alongside the normal-theory bootstrap intervals

compared to being single. Annual income levels $\geq \$ 35,000$ were associated with significant increases in utility compared to annual incomes less than $\$ 20,000$. Living alone, experiencing COVID-19-like symptoms not requiring hospitalization, and having a family member diagnosed with COVID-19 $(n=187)$ were significantly associated with lower utility. Self-reported fear of COVID-19's impact on personal health
(1-10 scale) was negatively and significantly correlated with utility.

Table 4 displays the post-lasso OLS regression median bootstrap estimates, bootstrap standard errors, bootstrap confidence intervals, and corresponding $E$-values. Estimates for predictors appearing in both the standard OLS and post-lasso OLS are largely similar. All additional predictors selected by 


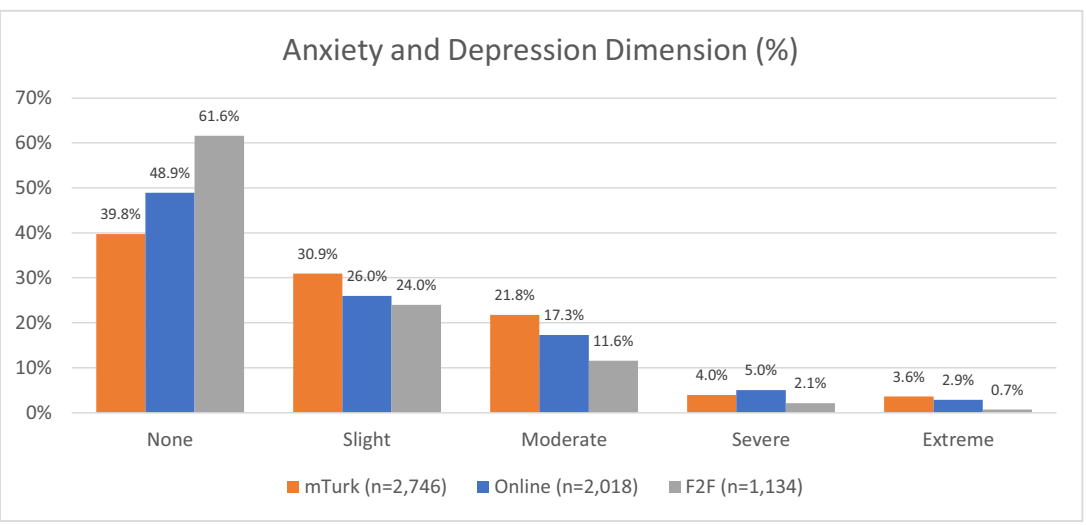

Figure 1 Anxiety and depression dimension. Percentage of individuals reporting none, slight, moderate, severe, and extreme problems with anxiety and depression by cohort. The during-pandemic cohort reports more problems than either online or face-to-face norms.

lasso - arthritis $(n=244)$, diabetes $(n=181)$, self-reported depression $(n=541)$, stroke $(n=28)$ interacted with fear of COVID-19's impact on health (1-10 scale), and underweight BMI $(n=120)$ interacted with residing in California ( $n=274)$ - are significantly associated with lower utility. As with gender, the significant interaction between underweight BMI and California residence is driven mainly by selfreported diagnosis of anxiety/depression, which is distinct from the EQ-5D question on anxiety/depression (Supplemental Figure 2).

Results of the $F$-test indicate the additional coefficients estimated by the post-lasso OLS significantly improve the model's ability to predict EQ-5D utility (see Technical Appendix). Based on the estimated variance-covariance matrix from the bootstrap estimates, we are confident that normal approximation for the coefficients is robust and inference is normal (Supplemental Table 1, Supplemental Figure 1). ${ }^{33}$

Post-lasso OLS estimates for annual income over $\$ 150,000$, arthritis, self-reported depression, and underweight BMI interacted with residing in California are most robust against bias from an unobserved confounder, all with $E$-value confidence limits $>2 .^{32}$ Relatively strong unmeasured confounding $(\mathrm{RR}>2.9)$ would be required to attenuate modeled effects.

\section{Population QALY Loss}

When extrapolated to the US population, we calculated an overall loss of 2.6 million QALYs compared to the prepandemic sample, a gain of 3.5 million QALYs compared to the online norm, and a loss of 8.4 million QALYs compared to the face-to-face norm. After dividing these values by life expectancy for each age group, we calculated an overall average gain of 18,385 lives at the expense of those aged 18-34. This was driven primarily by younger age groups, with average lives lost of 77,343 and 32,449 for 18-24 and 25-34 years old, respectively (Table 5).

\section{DISCUSSION}

HRQoL has decreased during COVID-19 compared to US population norms, especially for those aged $18-24$. This is unsurprising as the younger generation is likely more anxious about the future (education, career) and less firmly established in a set employment/career path. In addition, younger adults are at a critical life stage in developing and solidifying social relationships and networks, and social distancing/lockdowns due to COVID-19 have had a disproportionate impact on them, particularly as this age group is much less likely to be directly impacted by mortality due to COVID-19. These findings are similar to those using the EQ-5D-5L in Germany, which found members of the general public experienced worse HRQoL, particularly if they reported fear of COVID-19 or had a history of chronic illness and anxiety/depression ${ }^{19}$; similar EQ-5D findings in China also showed that lower income and unemployment decreased HRQoL. ${ }^{22,} 34$

Although HRQoL was higher than population norms among those $>35$ years, this may reflect a healthier, more highly educated sample compared to the US general population due to factors such as the ability to work from home without loss of pay, spending more time with family and friends, and more flexibility allowing time for non-jobrelated tasks. Nonetheless, results suggest that the mental health impact of COVID-19 is significant. It is difficult if not impossible to disentangle the positive and negative impact of these elements, and it is also important to acknowledge that relationship between these factors and HRQoL may change as the pandemic continues.

We employed traditional OLS regression alongside a lassoselected bootstrapped linear model to identify significant associations between respondent characteristics and HRQoL. As demonstrated by the $F$-test, the post-lasso model significantly improved fit over the standard model, indicating that lasso provides a more sophisticated method for testing specifications in high dimensional settings vs. stepwise selection, with the additional benefit of decreasing variance of estimates 
Table 5 Change in QALYs and Lives Affected

\begin{tabular}{|c|c|c|c|c|c|}
\hline \multicolumn{6}{|c|}{ Total change in QALYs } \\
\hline Age & US population & Pre & Online & F2F & Average \\
\hline $18-24$ & $31,678,500$ & $-5,340,457$ & $-2,917,051$ & $-5,292,939$ & $-4,516,816$ \\
\hline $25-34$ & $45,209,000$ & $-1,548,922$ & 649,879 & $-3,871,021$ & $-1,590,021$ \\
\hline $35-44$ & $41,027,000$ & $-920,509$ & $2,081,300$ & 153,031 & 437,940 \\
\hline $45-54$ & $40,700,000$ & $3,353,599$ & $2,366,624$ & 87,424 & $1,935,882$ \\
\hline $55-64$ & $41,755,000$ & $2,130,799$ & $1,483,597$ & 63,927 & $1,226,108$ \\
\hline$\geq 65$ & $52,787,000$ & $-225,999$ & $-208,403$ & 425,041 & $-3,120$ \\
\hline \multicolumn{2}{|r|}{ Total QALY change } & $-2,551,488$ & $3,455,945$ & $-8,434,537$ & $-2,510,027$ \\
\hline \multicolumn{6}{|c|}{ Total change in lives } \\
\hline Age & Life expectancy (years remaining) & Pre & Online & F2F & Average \\
\hline $18-24$ & 58.40 & $-91,446$ & $-49,950$ & $-90,633$ & $-77,343$ \\
\hline $25-34$ & 49.00 & $-31,611$ & 13,263 & $-79,000$ & $-32,449$ \\
\hline $35-44$ & 39.80 & $-23,128$ & 52,294 & 3,845 & 11,004 \\
\hline $45-54$ & 30.8 & 108,883 & 76,838 & 2,838 & 62,853 \\
\hline $55-64$ & 22.50 & 94,702 & 65,938 & 2,841 & 54,494 \\
\hline \multirow[t]{2}{*}{$\geq 65$} & 18 & $-12,555$ & $-11,578$ & 23,613 & -173 \\
\hline & Total lives affected & 44,845 & 146,805 & $-136,495$ & 18,385 \\
\hline
\end{tabular}

*F2F: face-to-face

compared to the standard model. We also calculated $E$-values for all point estimates and found that relatively strong unmeasured confounding $(R R \geq 2.9)$ would be required to attenuate all lasso-selected effects. For additional details, refer to the Technical Appendix.

We compared our results to a similar multivariable analysis of sociodemographic and behavioral predictors of EQ-5D utility based on a 2008 general population survey in England. ${ }^{35}$ While most predictors examined were included in our model, they also reported estimates for additional confounders such as alcohol consumption, smoking, fruit and vegetable intake, and physical activity. Among these, only being physically inactive has an effect size upper bound of $R R>2.5$. Given this, and the fact that we demonstrate good covariate control in our model, our estimates are robust to unobserved confounders like alcohol, smoking, diet, and exercise. Since the UK study is based on an EQ-5D-5L survey of the general population (much like ours) in England (culturally similar to the USA) and adjusts for most of the factors included in our regression, we find it is a good fit for contextualizing the $E$ values of our estimates.

A key limitation is that our sample was restricted to the online MTurk platform, which has been shown to have mixed external validity on context. ${ }^{28,}{ }^{36-39}$ Nonetheless, we believe our results overestimate HRQoL as MTurk workers are more likely to be those who have the flexibility to complete online tasks, and thus less likely to live and work in situations that would be heavily impacted by COVID-19, such as job loss or furlough. Nationally, reported unemployment rates reached a high of nearly $15 \%$ in April and have remained in double digits since, yet some metropolitan areas have reported numbers $>30 \%{ }^{40,41}$ These numbers are far higher than the $5.8 \%$ who reported job loss and the $9.6 \%$ who reported being temporarily laid off in our sample. As seen from the employment characteristics of our sample, respondents were more likely to work in jobs that can be done remotely. It is therefore likely that those who experience significant job loss and/or loss income, and thus more likely to report worse HRQoL, are not being adequately captured in our sample.

\section{CONCLUSION}

COVID-19's impact on American HRQoL varies by age group, with the largest negative impact on young adults aged 18-24 years. These results suggest that policies such as universal lockdowns, without risk-assessment by age or demographic characteristics, may have been implemented at the expense of the mental well-being of younger adults whose health outcomes have been discounted relative to older adults $>35$ years based on policy initiatives to date; under normal circumstances, this group (and particularly more elderly individuals) are more likely to stay home and self-isolate compared to younger adults; thus, lockdowns may have had a lower impact on this group than on younger adults, who are generally more mobile in their normal daily activities. It is important to consider the long-term implications of policies implemented during epidemics that may disproportionately impact the health and well-being of subgroups of the population, such as young adults in the USA during the COVID-19 pandemic.

Corresponding Author: Cynthia L. Gong, PharmD, PhD; Fetal \& Neonatal Institute, Children's Hospital Los Angeles, Los Angeles, CA, USA (e-mail: gongc@usc.edu).

Supplementary Information The online version contains supplementary material available at https://doi.org/10.1007/s11606-02106674-z.

Funding This study was funded by research grant \# EQ-84-2020-RA from the Euroßol Society. 


\section{Declarations:}

Conflict of Interest: ASP reports that he is a member of the Euroßol group and a partner in a health care consulting company, Second City Outcomes Research $L L C$, but that work has no bearing on the content of this manuscript. FX and NYG are also members of the Euroßol group. NKZ and SAC report grants from EuroBol Foundation during the conduct of the study. All other authors report no other conflicts of interest.

\section{REFERENCES}

1. Wan w. The coronavirus pandemic is pushing America into a mental health crisis. Washington Post. https://www.washingtonpost.com/ health/2020/05/04/mental-health-coronavirus/. Published May 4, 2020

2. Achenbach J. Coronavirus is harming the mental health of tens of millions of people in U.S., new poll finds. Washington Post. https:// www.washingtonpost.com/health/coronavirus-is-harming-the-mentalhealth-of-tens-of-millions-of-people-in-us-new-poll-finds/2020/04/02/ 565e6744-74ee-11ea-85cb-8670579b863d_story.html. Published April 2, 2020.

3. Petersen A. More People Are Taking Drugs for Anxiety and Insomnia, and Doctors Are Worried. The Wall Street Journal. https://www.wsj.com/ articles/more-people-are-taking-drugs-for-anxiety-and-insomnia-anddoctors-are-worried-11590411600. Published May 25, 2020.

4. Petersen A. The Struggle to Cope With Depression Amid Coronavirus. The Wall Street Journal. https://www.wsj.com/articles/the-struggle-tocope-with-depression-amid-coronavirus-11586696401. Published April 12, 2020.

5. Bettinger-Lopez C, Bro A. A Double Pandemic: Domestic Violence in the Age of COVID-19. Council on Foreign Relations. https://www.cfr.org/inbrief/double-pandemic-domestic-violence-age-covid-19. Published May 13, 2020.

6. Mohan M, Poidevin O Le. Coronavirus: Domestic violence "increases globally during lockdown.” BBC News. https://www.bbc.com/news/av/ world-53014211/coronavirus-domestic-violence-increases-globally-during-lockdown. Published June 12, 2020

7. Taub A. A New Covid-19 Crisis: Domestic Abuse Rises Worldwide. The New York Times. https://www.nytimes.com/2020/04/06/world/coronavirus-domestic-violence.html. Published April 14, 2020.

8. Hyland $\mathbf{P}$, Shevlin M, McBride $\mathbf{O}$, et al. Anxiety and depression in the Republic of Ireland during the COVID-19 pandemic. Acta Psychiatr Scand. Published online 2020. doi:10.1111/acps.13219

9. Guo $\mathbf{Y}$, Cheng $\mathbf{C}$, Zeng $\mathbf{Y}$, et al. Mental health disorders and associated risk factors in quarantined adults during the COVID-19 outbreak in China: a cross-sectional study. J Med Internet Res. Published online 2020. 10.2196/20328

10. Patsali ME, Mousa D-P V., Papadopoulou EVK, et al. University students' changes in mental health status and determinants of behavior during the COVID-19 lockdown in Greece. Psychiatry Res. Published online 2020:113298. https://doi.org/10.1016/j.psychres.2020.113298

11. Shader RI. COVID-19 and Depression. Clin Ther. 2020;(January). https://doi.org/10.1016/j.clinthera.2020.04.010

12. Pappa S, Ntella V, Giannakas T, Giannakoulis VG, Papoutsi E, Katsaounou P. Prevalence of depression, anxiety, and insomnia among healthcare workers during the COVID-19 pandemic: A systematic review and meta-analysis. Brain Behav Immun. Published online 2020. https:// doi.org/10.1016/j.bbi.2020.05.026

13. Skoda E-M, Teufel M, Stang A, et al. Psychological burden of healthcare professionals in Germany during the acute phase of the COVID-19 pandemic: differences and similarities in the international context. $J$ Public Health (Bangkok). Published online 2020. 10.1093/pubmed/ fdaal24

14. Fayers PM, Machin D. Quality of Life: The Assessment, Analysis and Interpretation of Patient-Reported Outcomes: Second Edition.; 2007. https://doi.org/10.1002/9780470024522

15. Euro@ol Society. EQ-5D-5L - About. Euro@ol.org. Published 2017. https://euroqol.org/eq-5d-instruments/eq-5d-5l-about/

16. Jiang R, Janssen B, Pickard A. PNS201 UNITED STATES POPULATION NORMS FOR THE EQ-5D-5L. Value Heal. 2020;23:S320-S321.
17. Pickard AS, Law EH, Jiang R, et al. United States Valuation of EQ-5D5L Health States Using an International Protocol. Value Heal. 2019;22(8):931-941. https://doi.org/10.1016/j.jval.2019.02.009

18. Cohen A, Selles RW, et al. What Is the Impact of the COVID-19 Pandemic on Quality of Life and Other Patient-reported Outcomes? An Analysis of the Hand-Wrist Study Cohort. Clin Orthop Relat Res. 2020;Publish Ahead of Print. https://doi.org/10.1097/corr. 0000000000001514

19. Bäuerle A, Steinbach J, Schweda A, et al. Mental Health Burden of the COVID-19 Outbreak in Germany: Predictors of Mental Health Impairment. J Prim Care Community Heal. 2020;11. https://doi.org/10.1177/ 2150132720953682

20. Garrigues E, Janvier $\mathbf{P}$, Kherabi Y, et al. Post-discharge persistent symptoms and health-related quality of life after hospitalization for COVID-19. J Infect. Published online 2020. https://doi.org/10.1016/j. jinf.2020.08.029

21. Azizi A, Achak D, Aboudi K, et al. Health-related quality of life and behavior-related lifestyle changes due to the COVID-19 home confinement: Dataset from a Moroccan sample. Data Br. 2020;32. https://doi. org/10.1016/j.dib.2020.106239

22. Ping $\mathbf{W}, \mathbf{Z h e n g} \mathbf{J}$, Niu $\mathbf{X}$, et al. Evaluation of health-related quality of life using EQ-5D in China during the COVID-19 pandemic. PLoS One. 2020;15(6). https://doi.org/10.1371/journal.pone.0234850

23. Wong EL-Y, Ho K-F, Wong SY-S, et al. Views on Workplace Policies and its Impact on Health-Related Quality of Life During Coronavirus Disease (COVID-19) Pandemic: Cross-Sectional Survey of Employees. Int J Heal Policy Manag. Published online 2020. https://doi.org/10.34172/ijhpm. 2020.127

24. Tran BX, Nguyen HT, Le HT, et al. Impact of COVID-19 on Economic Well-Being and Quality of Life of the Vietnamese During the National Social Distancing. Front Psychol. 2020;11. https://doi.org/10.3389/ fpsyg.2020.565153

25. Lara B, Carnes A, Dakterzada F, Benitez I, Piñol-Ripoll G. Neuropsychiatric symptoms and quality of life in Spanish patients with Alzheimer's disease during the COVID-19 lockdown. Eur J Neurol. 2020;27(9):17441747. https://doi.org/10.1111/ene.14339

26. Amazon Mechanical Turk. Accessed June 9, 2020. https://www.mturk. com/worker/help\#what_is

27. Shapiro DN, Chandler J, Mueller PA. Using mechanical turk to study clinical populations. Clin Psychol Sci. 2013;1(2):213-220. https://doi. org/10.1177/2167702612469015

28. Mortensen K, Alcalá MG, French MT, Hu T. Self-reported Health Status Differs for Amazon's Mechanical Turk Respondents Compared with Nationally Representative Surveys. Med Care. 2018;56(3):211-215. https://doi.org/10.1097/MLR.0000000000000871

29. Tibshirani R. Regression Shrinkage and Selection via the Lasso. J R Stat Soc. 1996;58(1):267-288. https://doi.org/10.1017/ s0272503700054525

30. T H, Tibshirani R, Friedman J. The Elements of Statistical Learning: Data Mining, Inference, and Prediction. 2nd ed. Springer; 2009. 10.1007/ 978-1-4419-9863-7_941

31. VanderWeele $\mathbf{T} \boldsymbol{J}$, Ding $\mathbf{P}$. Sensitivity analysis in observational research: Introducing the E-Value. Ann Intern Med. 2017;167(4):268-274. https:// doi.org/10.7326/M16-2607

32. Haneuse $\mathbf{S}$, VanderWeele TJ, Arterburn D. Using the E-Value to Assess the Potential Effect of Unmeasured Confounding in Observational Studies. JAMA - J Am Med Assoc. 2019;321(6):602-603.

33. Fox J, Weisberg $\mathbf{S}$. Bootstrapping Regression Models in R. In: An $R$ Companion to Applied Regression, Third Edition. 3rd ed. SAGE Publications; 2019. https://socialsciences.mcmaster.ca/jfox/Books/Companion/appendices/Appendix-Bootstrapping.pdf

34. Guo $\mathbf{Y}$, Shen $\mathbf{M}$, Zhang $\mathbf{X}$, et al. Association of socioeconomic changes due to the COVID-19 pandemic with health outcomes in patients with skin diseases: Cross-sectional survey study. J Med Internet Res. 2020;22(9). https://doi.org/10.2196/22288

35. Maheswaran H, Petrou S, Rees K, Stranges S. Estimating EQ-5D utility values for major health behavioural risk factors in England. J Epidemiol Community Health. 2013;67(2):172-180. https://doi.org/10.1136/jech2012-201019

36. Huff C, Tingley D. "Who are these people?" Evaluating the demographic characteristics and political preferences of MTurk survey respondents. Res Polit. 2015;2(3). https://doi.org/10.1177/2053168015604648

37. Buhrmester M, Kwang T, Gosling SD. Amazon's mechanical Turk: A new source of inexpensive, yet high-quality, data? Perspect Psychol Sci. 2011;6(1):3-5. https://doi.org/10.1177/1745691610393980 
38. Walters K, Christakis DA, Wright DR. Are Mechanical Turk worker samples representative of health status and health behaviors in the U.S.? PLoS One. 2018;13(6). https://doi.org/10.1371/journal.pone.0198835

39. Berinsky AJ, Huber GA, Lenz GS. Evaluating online labor markets for experimental research: Amazon.com's mechanical turk. Polit Anal 2012;20(3):351-368. https://doi.org/10.1093/pan/mpr057

40. US Bureau of Labor Statistics. Unemployment Rates for States, Seasonally Adjusted. Local Area Unemployment Statistics. Published 2020 Accessed July 28, 2020. https://www.bls.gov/web/laus/laumstrk.htm

41. US Bureau of Labor Statistics. Over-the-Year Change in Unemployment Rates for Metropolitan Areas, Not Seasonally Adjusted. Local Area Unemployment Statistics. Published 2020. Accessed July 28, 2020. https://www.bls.gov/web/metro/laummtch.htm

42. US Census Bureau. Age and Sex Composition in the United States: 2019, Table 1. Population by Age and Sex. Published 2019. Accessed July 28 2020. http://www.census.gov/population/age/data/files/2012/ 2012gender_table1.xlsx

43. US Census Bureau. QuickFacts United States V2019. Published 2019. Accessed July 28, 2020. https://www.census.gov/quickfacts/fact/table/ US/PST045219

44. US Census Bureau. Educational Attainment in the United States: 2019 Table 1. Educational Attainment of the Population 18 Years and Over, by Age, Sex, Race, and Hispanic Origin: 2019. Published 2019. Accessed July 28, 2020. https://www.census.gov/data/tables/2019/demo/educational-attainment/cps-detailed-tables.html

45. US Census Bureau. Marital Status, Table ID: S1201. American Community Survey. Published 2019. Accessed July 28, 2020. https://data census.gov / cedsci $/$ table $? q=$ marriage $\&$ hidePreview $=$ false $\&$ ti $\mathrm{d}=$ ACSST1Y2018.S1201\&vintage $=2018$

46. US Census Bureau. HINC-01. Selected Characteristics of Households by Total Money Income. Current Population Survey (CPS) Annual Social and Economic (ASEC) Supplement. Published 2018. Accessed July 28, 2020 https://www.census.gov/data/tables/time-series/demo/income-poverty/cps-hinc/hinc-01.html

47. Kaiser Family Foundation. Health Insurance Coverage of the Total Population. State Health Facts. Published 2018. Accessed July 28, 2020. https://www.kff.org/other/state-indicator/totalpopulation/?current-Timeframe $=0$ \& s ort Model=\% 7 B $\% 22$ colId $\% 22: \% 22$ ?Location $\% 22, \% 22$ sort $\% 22: \% 22$ asc $\% 22 \% 7 \mathrm{D}$

48. Berchick ER, Barnett JC, Upton RD. Health Insurance Coverage in the United States: 2018. US Census Bureau REPORT NUMBER P60-267 (RV). Published 2019. Accessed July 28, 2020. https://www.census.gov/ library/publications/2019/demo/p60-267.html

49. Gallup. Party Affiliation. IN DEPTH: TOPICS A TO Z. Published 2020. Accessed July 28, 2020. https://news.gallup.com/poll/15370/partyaffiliation.aspx

50. US Census Bureau. U.S. Census Bureau Releases 2018 Families and Living Arrangements Tables. RELEASE NUMBER CB18-TPS.54. Published 2018. Accessed July 28, 2020. https://www.census.gov/newsroom/press-releases/2018/families.html

51. Centers for Disease Control and Prevention. High Cholesterol Statistical Reports for Health Professionals. High Cholesterol Statistics and Maps. Published 2017. Accessed July 28, 2020. https://www.cdc.gov/cholesterol/statistical_reports.htm

52. Centers for Disease Control and Prevention. Hypertension. National Center for Health Statistics. Published 2018. Accessed July 28, 2020 https://www.cdc.gov/nchs/fastats/hypertension.htm

53. Centers for Disease Control and Prevention. Arthritis. National Center for Health Statistics. Published 2018. Accessed July 28, 2020. https://www. cdc.gov/nchs/fastats/arthritis.htm

54. Centers for Disease Control and Prevention. Diabetes. National Diabetes Statistics Report. Published 2020. Accessed July 28, 2020. https://www. cdc.gov/diabetes/data/statistics/statistics-report.html

55. Centers for Disease Control and Prevention. Heart Disease. Patient Education Handouts. Published 2019. Accessed July 28, 2020. https:// www.cdc.gov/heartdisease/heart_failure.htm

56. Centers for Disease Control and Prevention. Cerebrovascular Disease or Stroke. National Center for Health Statistics. Published 2018. Accessed July 28, 2020. https://www.cdc.gov/nchs/fastats/stroke.htm

57. Centers for Disease Control and Prevention. Chronic Obstructive Pulmonary Disease (COPD) Includes: Chronic Bronchitis and Emphysema. National Center for Health Statistics. Published 2018. Accessed July 28, 2020. https://www.cdc.gov/nchs/fastats/copd.htm

58. Centers for Disease Control and Prevention. Asthma. National Center for Health Statistics. Published 2018. Accessed July 28, 2020. https://www. cdc.gov/nchs/fastats/asthma.htm

59. Centers for Disease Control and Prevention. Depression. National Center for Health Statistics. Published 2017. Accessed July 28, 2020. https:// www.cdc.gov/nchs/fastats/depression.htm

60. Centers for Disease Control and Prevention. Summary Health Statistics: National Health Interview Survey, 2018 Table A-5a. Age-Adjusted Percentages (with Standard Errors) of Migraines and Pain in Neck, Lower Back, Face, or Jaw among Adults Aged 18 and over, by Selected Characteristics: United States.; 2018. https://ftp.cdc.gov/pub/Health_Statistics/NCHS/ NHIS/SHS/2018_SHS_Table_A-5.pdf

61. Centers for Disease Control and Prevention. Cancer. National Center for Health Statistics. Published 2018. Accessed July 28, 2020. https://www. cdc.gov/nchs/fastats/cancer.htm

62. Centers for Disease Control and Prevention. Smoking \& Tobacco Use. Data and Statistics: Fast Facts. Published 2020. Accessed July 28, 2020. https://www.cdc.gov/tobacco/data_statistics/fact_sheets/fast_facts/index.htm

63. Centers for Disease Control and Prevention. Summary Health Statistics: National Health Interview Survey, 2017 Table A-12a. Age-Adjusted Percentages (with Standard Errors) of Current Cigarette Smoking Status among Adults Aged 18 and over, by Selected Characteristics: United States, 2017.; 2017. https://ftp.cdc.gov/pub/Health_Statistics/NCHS/ NHIS/SHS/2017_SHS_Table_A-12.pdf

64. DQYDJ. BMI Percentile Calculator: Body Mass Indexes in the United States. Published 2019. Accessed July 28, 2020. https://dqydj.com/ bmi-percentile-calculator-united-states/

65. Centers for Disease Control and Prevention. National Health and Nutrition Examination Survey 2015-2016. National Center for Health Statistics. Published 2020. Accessed July 28, 2020. https://wwwn.cdc. gov/nchs/nhanes/continuousnhanes/default.aspx?BeginYear=2015

66. US Census Bureau. Employment Status, TableID: S2301. American Community Survey. Published 2018. Accessed July 28, 2020. https:// data.census.gov/cedsci/table?q=s2301\&tid=ACSST1Y2018.S2301

Publisher's Note: Springer Nature remains neutral with regard to jurisdictional claims in published maps and institutional affiliations. 